\title{
NONMODAL CASCADE IN THE COMPRESSIBLE SOLAR ATMOSPHERE: SELF-HEATING, AN ALTERNATIVE WAY TO ENHANCE WAVE HEATING
}

\author{
Bidzina M. Shergelashvili, ${ }^{1,2}$ Stefaan Poedts, ${ }^{1}$ and Avtandil D. Pataraya ${ }^{2}$ \\ Received 2005 June 22; accepted 2006 March 17; published 2006 April 11
}

\begin{abstract}
The nonmodal self-heating mechanism recently proposed by Rogava is applied to the medium of solar coronal holes with an inhomogeneous plasma flow along the magnetic field lines. The viscosity force is assumed to be anisotropic. The efficiency of the nonmodal cascade process is examined for different sets of environmental parameters and for different wave parameters. It is concluded that the proposed mechanism can serve as an alternative mechanism for explaining the significant heat production in the lower corona, even when only laminar values of the viscosity coefficients are taken into account.
\end{abstract}

Subject headings: MHD — Sun: corona — waves

\section{INTRODUCTION}

The dissipation of MHD waves in the solar atmosphere is a key issue in the coronal heating problem. It is well known that the dynamics of the coronal plasma is predominantly governed by the magnetic field, leading to the anisotropic nature of the transport processes. All mechanisms for wave heating of the corona face the same problem (Aschwanden 2004): the characteristic spatial scales of the coronal wave phenomena are relatively large, so their effective dissipation requires some mechanism to transform these large length scales into much smaller ones. Alfvén waves, for example, are only affected by the "shear" viscosity because of the purely transverse and incompressible nature of these modes. It is estimated that this shear component of the viscosity tensor in the anisotropic plasma of the solar atmosphere is about 10 orders of magnitude smaller than the bulk viscosity. Therefore, Alfvén disturbances turn out to be almost nondissipative unless some dynamical mechanisms are available either for a significant reduction of their spatial scales or for a substantial transformation of their oscillatory nature from incompressible to compressible.

A well-known mechanism for reducing the spatial scales of waves is phase mixing, for example, the phase mixing of Alfvén waves due to inhomogeneous magnetic field or density profiles (Heyvaerts \& Priest 1983). Recently, the efficiency of phase mixing of Alfvén waves has been studied in the open magnetic field line configurations of the solar corona by Ruderman et al. (1998). These authors considered a two-dimensional model of a coronal hole and studied the problem by means of a WKB approach. The effect of stationary flows on the process of phase mixing in the solar atmosphere has also been studied in the past (Ryutova \& Habbal 1995). However, the dissipation of MHD waves and their role in the coronal heating problem has, so far, not been addressed by means of their nonmodal evolution in time (the nonmodal coupling of MHD waves in the solar wind has been studied by Poedts et al. [1998]).

In this Letter, we present an alternative mechanism for enhancing the wave heating process in the solar corona. This alternative mechanism is associated with the inhomogeneity of the background flow. The "self-heating mechanism" (Rogava

\footnotetext{
${ }^{1}$ Centrum voor Plasma-Astrophysica, Katholieke Universiteit Leuven, Celestijnenlaan 200B, B-3001 Heverlee, Belgium; Bidzina.Shergelashvili@ wis.kuleuven.be, Stefaan.Poedts@wis.kuleuven.be.

${ }^{2}$ Center for Plasma Astrophysics, Georgian National Astrophysical Observatory, 2a, Kazbegi Avenue, 0160 Tbilisi, Georgia.
}

2004) is related to the nonmodal behavior of the linear perturbations of the MHD system with an inhomogeneous background flow, which can coexist and operate independently in the solar corona. Here we address only the simplest case, an equilibrium outward velocity field directed along the equilibrium magnetic field with a linear shear profile across this field. In the real situation the flow can have a more complex (e.g., helical) nature, leading to even more intense heat production. The mechanism applied was suggested recently by Rogava (2004, where the process is studied for acoustic waves in hydrodynamic fluid flows), and a preliminary study of its efficiency in the magnetic structures in the solar atmosphere is given in Shergelashvili et al. (2004).

The self-heating process may have far-reaching consequences for the long-standing problem of solar coronal heating, as it introduces the idea that the source of the heating may be not only directly excited Alfvén waves (due to the photospheric convective motions), as widely assumed in the solar physics community, but also the compressional fast magnetosonic waves excited and amplified by the background plasma flow in the corona itself. The basic idea is that the waves act as transmitters of the shearing energy of the flow into heat.

\section{THE BASIC MODEL}

We aim to study the evolution of waves in the nonuniform, "open" magnetic field configurations in the solar corona, called coronal holes. It is known that these parts of the solar atmosphere consist of plasmas with inhomogeneous flow velocities and density profiles and that the dynamics of these regions is predominantly governed by their nonuniform, almost vertical magnetic fields. A two-dimensional model of such an atmospheric structure, well illustrating its basic properties, can be found in Ruderman et al. (1998). These authors studied the dissipation of Alfvén waves due to phase mixing. In general, when the propagation of MHD waves in a nonuniform plasma is considered, one should identify the spatial scales of the inhomogeneity of the physical background quantities and their relative importance in the concrete configuration.

We intend to investigate the evolution of Alfvén waves in a compressional, magnetically dominated medium with a nonuniform plane background flow $\boldsymbol{U}_{0}=\left(0,0, U_{0 z}(x)\right)=(0,0, A x)$ (the constant $A$ denotes the velocity shear rate) directed outward, along the uniform magnetic field lines $\boldsymbol{B}_{0}=\left(0,0, B_{0}\right)$. The chosen model configuration for this study is justified by the considered characteristic length scales of the nonuniformi- 

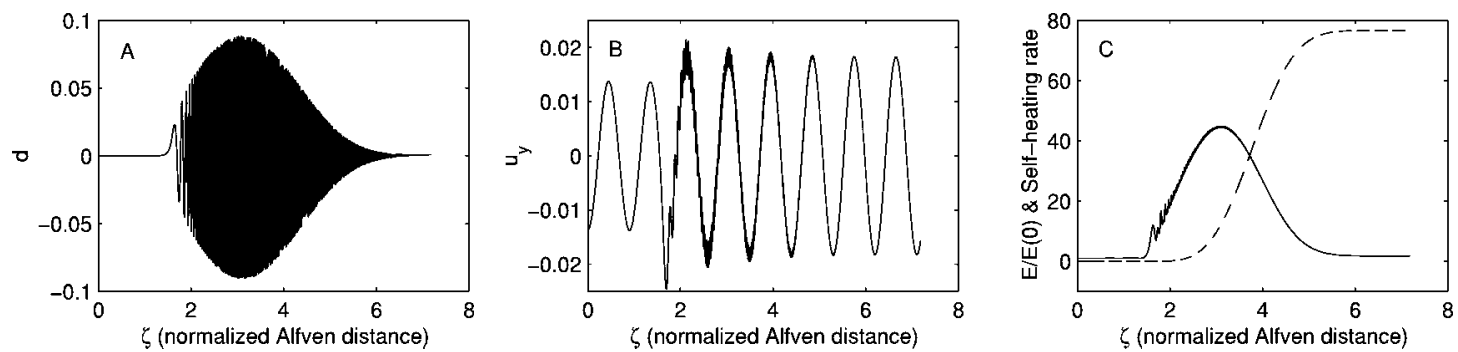

FIG. 1.-Plots of different physical quantities against the dimensionless time variable $\zeta=V_{\mathrm{A}} t / R_{\odot}$, which represents the Alfvén distance $\left(V_{\mathrm{A}} t\right)$ in units of the solar radius. (a) Normalized density perturbation $d ;(b)$ dimensionless $y$-component of the velocity perturbation, $u_{v} ;(c)$ total perturbation energy normalized by its initial value (solid line) and the self-heating function (eq. [7]) (dashed line).

ties (in this case, in the direction transverse to the magnetic field) of the different physical quantities $f$, defined as

$$
\frac{1}{L_{f}}=\frac{1}{f} \frac{d f}{d x}
$$

We deal with those parts of the coronal holes where the flow velocity gradients are so strong that

$$
L_{u} \ll L_{B}, \quad L_{u} \ll L_{\rho} .
$$

Hence, the effects of the nonuniformity of $B_{0}$ and $\rho_{0}$ can be omitted (for a similar discussion in the context of laboratory plasmas, see Mikhailenko et al. 2002). Our interest is to find the solution of an initial-value problem constructed through the linearization of the basic set of MHD equations. In order to handle this problem, we follow the standard nonmodal formalism.

The nonmodal analysis is a mathematical method that transforms the spatial dependence related to the sheared background flow into a temporal dependence, so that the problem can be treated as an initial-value problem. In the particular case of the background flow profile we are addressing here, the $k_{y}$ and $k_{z}$ components of the wavevector are constants, while $k_{x}=$ $k_{x 0}-A k_{z} t$. With this notation, the governing set of (temporally inhomogeneous) equations takes the following dimensionless form:

$$
\begin{gathered}
d^{(1)}=\boldsymbol{K} \cdot \boldsymbol{u}, \\
\boldsymbol{u}^{(1)}+R u_{x} \boldsymbol{e}_{z}=-\boldsymbol{K} \epsilon^{2} d+\boldsymbol{b}-\boldsymbol{K} b_{z}+\boldsymbol{F}, \\
b_{x}^{(1)}=-u_{x}, \quad b_{y}^{(1)}=-u_{y}, \\
\boldsymbol{K} \cdot \boldsymbol{b}=0,
\end{gathered}
$$

where the superscript "(1)" denotes the derivative with respect to the dimensionless time derivative $\tau=V_{\mathrm{A}} k_{z} t ; \boldsymbol{K}=\left(K_{x}, K_{y}, 1\right)$ with $K_{x}=k_{x} / k_{z}$ and $K_{y}=k_{y} / k_{z}$; and $u_{x}=u_{x}^{\prime} / V_{\mathrm{A}}, u_{y}=u_{y}^{\prime} / V_{\mathrm{A}}$, and $u_{z}=u_{z}^{\prime} / V_{\mathrm{A}}$ and $b_{x}=i b_{x}^{\prime} / B_{0}, b_{y}=i b_{y}^{\prime} / B_{0}$, and $b_{z}=i b_{z}^{\prime} / B_{0}$ are the dimensionless components of the velocity perturbation and the magnetic field perturbation, respectively, while $\boldsymbol{e}_{z}$ is the unit vector along the $z$-direction. In addition, $R=A / k_{z} V_{\mathrm{A}}$ and $F_{x}, F_{y}$, and $F_{z}$ are the components of the viscous force. Finally, $d=i \rho^{\prime} / \rho_{0}$ denotes the dimensionless plasma density and $\epsilon=C_{s} / V_{\mathrm{A}}$. We consider the case of a strong magnetic field so that the viscosity force is very anisotropic. Therefore, we write the components of the viscous tensor following Braginskii (1965) in terms of the stress tensor. We also introduce here the dimensionless total perturbation energy $E=\epsilon^{2} d^{2} / 2+u^{2} / 2+$ $b^{2} / 2$. We define the self-heating function in a similar manner to Rogava (2004) and Shergelashvili et al. (2004):

$$
\Lambda(t) \equiv \frac{1}{E(0)} \int_{0}^{t}(\boldsymbol{F} \cdot \boldsymbol{u}) d t^{\prime}
$$

with the total self-heating rate $\Lambda_{\infty} \equiv \lim _{t \rightarrow \infty} \Lambda(t)$. In order to study the evolution of the spatial Fourier harmonics in time and their interaction with each other and with the background flow, we need to solve the system of equations (3)-(6). This set of equations governs the linear dynamics of the Alfvén and the fast and slow magnetoacoustic MHD waves, their nonmodal coupling by the shear flow, and the rates of their viscous dissipation. We emphasize the cascade nature of the process because a given perturbation passes through few stages during the time starting with its excitation and ending with its dissipation.

\subsection{Results}

We solved the set of equations (3)-(6) numerically. We considered input parameter values that are typical for the open magnetic field line structures of the corona. We assume that, initially, a purely incompressible Alfvén wave disturbance is excited. The results of our calculations for a given set of input parameters are shown in Figure 1. For determining the characteristic value of the transverse inhomogeneity length scales of the magnetic field and the density, we follow the work by Ruderman et al. (1998), $L_{B}=L_{\rho}=H_{\perp} \approx 5 \mathrm{Mm}$. Further, we estimate the value of $L_{u}=u_{0} / A$, where $u_{0}$ is the characteristic value of the velocity, which is found to be much less than $V_{\mathrm{A}}$ in the lower corona (see Nakariakov et al. 1998). If we consider the areas of the coronal holes where the velocity of the plasma macroscopic flow varies between 5 and $40 \mathrm{~km} \mathrm{~s}^{-1}$, then inequality (2) holds when $A \gg u_{0} / H_{\perp}$, yielding $A \gg 10^{-3} \mathrm{~s}^{-1}$ (for $u_{0}=5 \mathrm{~km} \mathrm{~s}^{-1}$ ) and $A \gg 8 \times 10^{-3} \mathrm{~s}^{-1}$ (for $u_{0}=40 \mathrm{~km} \mathrm{~s}^{-1}$ ). For larger values of $H_{\perp}$, the thresholds can be even smaller. In our calculation, we use the value $A=0.05 \mathrm{~s}^{-1}$ for the velocity shear rate. Other parameter values used in these calculations are the following: the components of the viscosity tensor are $\nu_{0}=10^{14} \mathrm{~cm}^{2} \mathrm{~s}^{-1}$ (compressional), $\nu_{1}=\nu_{2}=10^{4} \mathrm{~cm}^{2} \mathrm{~s}^{-1}$ (shear), and $\nu_{3}=\nu_{4}=10^{9} \mathrm{~cm}^{2} \mathrm{~s}^{-1} ; K_{x}(0)=55, K_{y}(0)=1$, and $k_{z}=10^{-10} \mathrm{~cm}^{-1} ; \epsilon=0.1 ; V_{\mathrm{A}}=1000 \mathrm{~km} \mathrm{~s}^{-1} ; C=$ $100 \mathrm{~km} \mathrm{~s}^{-1}$; and the Alfvén wave period $T \approx 10.5$ minutes. Initially, we have a purely transverse $\boldsymbol{K} \cdot \boldsymbol{u}(0)=0$ disturbance without a perturbation of the density (see Fig. 1a). The initial conditions are $u_{x}(0) V_{\mathrm{A}}=2.5 \times 10^{-4} V_{\mathrm{A}}=0.25 \mathrm{~km} \mathrm{~s}^{-1}$ and $u_{y}(0) V_{\mathrm{A}}$ $=-\left[K_{x}(0) u_{x}(0) / K_{y}\right] V_{\mathrm{A}}=-1.38 \times 10^{-2} V_{\mathrm{A}}=-13.75 \mathrm{~km} \mathrm{~s}^{-1}$. 
All other perturbation quantities are set to zero at $t=0$. In Figure 1, we show the results for an Alfvén wave with an initial wavelength of $\approx 6.3 \times 10^{10} \mathrm{~cm}$. This appears to be the maximum value of the modal spatial scale, for which the self-heating cascade ends within a typical distance of 5 solar radii. We can distinguish the following stages in the temporal evolution of the system:

1. At first, the perturbation consists only of the initial transverse Alfvén wave, which is weakly damped because only the shear viscosity is involved in the damping process at this stage. During this phase, $k_{x}$ decreases slowly with time (as $\left.k_{x}=k_{x 0}-A k_{z} t\right)$.

2. In the "degenerate area," that is, in the time interval when $k_{x}(t) \approx 0$ (for the case shown in Fig. 1 , this is the interval $1050 \mathrm{~s} \lesssim t \lesssim 1200 \mathrm{~s}$, with $\Delta t=150 \mathrm{~s}<T$ ), the background flow couples the Alfvén wave with the compressible fast magnetoacoustic wave, which satisfies specific resonant conditions in this time interval (for details, see, e.g., Poedts et al. 1998; Rogava et al. 2000; references therein). As a result, the fast wave is excited at this stage and the oscillatory signal shown in Figure 1 becomes a mixture of two signals corresponding to the Alfvén and fast magnetoacoustic perturbations, respectively.

3. Outside the resonant area, the nonmodal coupling of waves becomes negligible and the Alfvén and fast magnetoacoustic waves start to evolve in time independently of each other. The Alfvén wave continues to propagate freely with a somewhat adjusted energy after the coupling. While the justexcited fast magnetoacoustic wave gains energy from the background flow its wavelength becomes shorter, as $\left|k_{x}(t)\right|$ now increases in time.

4. In the final stage, the wavelength of the fast magnetoacoustic wave becomes so small that the viscous dissipation becomes prevalent and rapidly dissipates this wave, transforming a significant amount of energy from the wave (including the part it gained from the background flow) into heat. This amount of energy can be many times the initial energy of the perturbation (for the case shown, $\Lambda_{\infty} \approx 80$ ). Therefore, as is seen in Figure 1, only the Alfvén disturbance survives this stage, unless of course any other mechanism directly acting on the Alfvén wave is involved in the process.

In general, the efficiency of the self-heating mechanism depends on the initial conditions and initial wavelength of the Alfvén wave. We also obtained a solution for another set of solar coronal parameter values corresponding to the approximately minimal wavelength $\approx 5.25 \times 10^{9} \mathrm{~cm}($ period $T \approx 50 \mathrm{~s})$, for which the self-heating generated heat amounts to at least 3 times the initial wave energy (i.e., $\Lambda_{\infty}>3$ ).

\section{DISCUSSION AND CONCLUSIONS}

We have studied the efficiency of the nonmodal cascade process known as "self-heating" of a nonuniform flow and its possible role in the heating of the solar corona and, in particular, of coronal holes. Our general analysis reveals that the shearing energy of the background flow can be an alternative energy "supplier" for the heating of the corona. The considered mechanism can operate in the solar atmosphere independently from (and thus simultaneously with) other mechanisms of wave dissipation. Some important aspects of the results obtained are given below.

The solar coronal holes include remarkable nonuniform magnetic structures and flows. Therefore, under certain con- ditions different mechanisms of MHD wave damping can dominate or cooperate along with other mechanisms. As we have defined the measure of the nonuniformity length scale by inequality (2), we can identify different scenarios for the dissipation of waves in the solar atmosphere:

1. Within the areas of the solar coronal holes where $L_{u} \gg$ $L_{B}$ and $L_{u} \gg L_{\rho}$, the phase mixing of Alfvén waves is the dominant mechanism. As a result, the effect of the flow velocity gradients can be neglected in these regions.

2. In the regions where $L_{u} \sim L_{B}$ and $L_{u} \sim L_{\rho}$, the characteristic length scales of the inhomogeneities are of the same order of magnitude. In these areas, phase mixing and the effect of nonmodal self-heating can be of comparable importance, under favorable conditions. However, these two mechanisms still can operate "separately" in the atmosphere without contradicting each other. In general, the operation of the selfheating mechanism in a medium with spatially anisotropic transport processes does not require the dissipation of the initial Alfvén wave. Instead, at a certain moment the fast compressional mode can be excited, which then evolves and dissipates independently, while the Alfvén wave itself can continue to be damped by the phase mixing. In order to arrive at a more quantitative picture of this scenario, an analytical model, similar to the one given by Ruderman et al. (1998), should be developed. This would describe the Alfvén wave phase mixing in the nonmodal approach (i.e., as an initial-value problem). This, however, is beyond the scope of the current study and might become the subject of a subsequent investigation in the near future.

3. Within the areas where $L_{u} \ll L_{B}$ and $L_{u} \ll L_{\rho}$, the flow velocity gradients are strong enough to predominantly govern the linear wave dynamics of the system. The nonuniformities of the magnetic field and density can then be safely neglected, and one can study the nonmodal self-heating process separately. This is what we did in the present Letter. We found that the considered cascade processes can lead to a substantial enhancement of the wave heating in the solar coronal holes.

4. Ruderman et al. (1998) have shown that for some choices of the magnetic field and density profiles, the phase mixing of the Alfvén waves works up to heights of a few scale heights $H$. Above this height, the mechanism becomes unimportant. At this stage, however, the self-heating cascade may be "turned on" and the effects of the nonmodal wave coupling, the reduction of the wave length, and the consequent dissipation of the waves can start dominating the behavior of the system.

The mechanism proposed in this Letter is of importance for the enhancement of the wave heating processes in the corona even without turbulent intensification of the dissipation processes, that is, when only the well-known laminar values of the viscosity coefficients are taken into account. It solves two problems at once, viz., (1) the energy source can be the background plasma flow instead of the subphotospheric convective motions and (2) the waves are generated and strongly amplified in the corona itself, so that there is no need for a transport mechanism to bring the energy from the photosphere through the transition region to the corona. It should be noted that there also exists a mechanism of direct nonlinear coupling of these waves in the nonuniform flow (Nakariakov et al. 1998), which could also be considered in a similar way. The process proposed in current Letter may be relevant for other stars as well and, in fact, for all flowing astrophysical plasmas. 
This work has been developed in the framework of the doctoral program of B. M. S. at the Centrum voor Plasma-Astrofysica, Katholike Universiteit Leuven (scholarships OE/04/33OE/05/18) and the projects GOA 2004/01 (K.U. Leuven) and
90203 (ESA PRODEX 8). We thank A. D. Rogava for many valuable discussions. B. M. S. is grateful to V. M. Nakariakov for informative discussions and suggestions. We thank the anonymous referee for constructive comments on our manuscript.

\section{REFERENCES}

Aschwanden, M. 2004, Physics of the Solar Corona (Berlin: Springer)

Braginskii, S. I. 1965, Rev. Plasma Phys., 1, 205

Heyvaerts, J., \& Priest, E. R. 1983, A\&A, 117, 220

Mikhailenko, V. S., Mikhailenko, V. V., Heyn, M. F., \& Mahajan, S. M. 2002, Phys. Rev. E, 66, 066409

Nakariakov, V. M., Roberts, B., \& Murawski, K. 1998, A\&A, 332, 795

Poedts, S., Rogava, A. D., Mahajan, S. M. 1998, ApJ, 505, 369
Rogava, A. D. 2004, Ap\&SS, 293, 189

Rogava, A. D., Poedts, S., \& Mahajan, S. M. 2000, A\&A, 354, 749

Ruderman, M. S., Nakariakov, V. M., \& Roberts, B. 1998, A\&A, 338, 1118

Ryutova, M. P., \& Habbal, S. R. 1995, ApJ, 451, 381

Shergelashvili, B. M., Rogava, A. D., \& Poedts, S. 2004, in Proc. SOHO 15 Workshop: Coronal Heating, ed. R. W. Walsh et al. (ESA SP-575) (Noordwijk: ESA), 437 